\title{
Abstract: Robust Multi-Scale Anatomical Landmark Detection in Incomplete 3D-CT Data
}

\author{
Florin C. Ghesu ${ }^{1,2}$, Bogdan Georgescu ${ }^{1}$, Sasa Grbic ${ }^{1}$, Andreas Maier $^{2}$, \\ Joachim Hornegger ${ }^{2}$, Dorin Comaniciu ${ }^{1}$ \\ ${ }^{1}$ Siemens Healthineers, Medical Imaging Technologies, Princeton, NJ, USA \\ ${ }^{2}$ Pattern Recognition Lab, Friedrich-Alexander-Universität, Erlangen, Germany \\ florin.c.ghesu@fau.de
}

An essential prerequisite for comprehensive medical image analysis is the robust and fast detection of anatomical structures in the human body. To this point, machine learning techniques are most often applied to address this problem, exploiting large annotated image databases to estimate parametric models for anatomy appearance. However, the performance of these methods is generally limited, due to suboptimal and exhaustive search strategies applied on large volumetric image data, e.g., 3D-CT scans. Most importantly, these techniques do not effectively address cases of incomplete data, i.e., scans taken with a partial field-of-view. We address these challenges by unifying the anatomy appearance model and the search strategy in the form of a behavior-learning task. This is solved using the capabilities of deep reinforcement learning with multi-scale image analysis and robust statistical shape modeling [1]. These mechanisms enable the teaching of intelligent artificial agents to find and follow optimal navigation paths in the image scale-space, that converge to the locations of target anatomical structures. At the same time, such navigation paths can account for missing structures to ensure the robust and spatially-coherent detection of the observed anatomical landmarks. Finally, we show how the identified landmarks can be used as robust guidance in estimating the extent of the body-region, captured by the CT scan. Experiments demonstrate that our solution outperforms a state-of-the-art deep learning method in detecting different anatomical structures, without any failure, on a dataset of over 2300 3D-CT volumes. In particular, our method achieves $0 \%$ false-positive and $0 \%$ false-negative rates at detecting the landmarks or recognizing their absence from the field-of-view of the scan. Most importantly, the detection-time of the reference method is reduced by $15-20$ times to under $40 \mathrm{~ms}$, an unmatched real-time performance for large 3D-CT data.

\section{References}

1. Ghesu FC, Georgescu B, Grbic S, et al. Robust multi-scale anatomical landmark detection in incomplete 3D-CT data. Proc MICCAI. 2017;Part I:194-202. 